\title{
ON ELECTRONIC STRUCTURE AND MAGNETIC BEHAVIOUR OF FCC IRON FILMS EPITAXIALLY GROWN ON $\mathrm{Cu}(001)$
}

\author{
R. WOJNECKI ${ }^{a *}$ AND A. SUKIENNICKI ${ }^{a, b}$ \\ anstitute of Physics, Polish Academy of Sciences \\ Al. Lotników 32/46, 02-668 Warsaw, Poland \\ ${ }^{b}$ Institute of Physics, Warsaw University of Technology \\ Koszykowa 75, 00-662 Warsaw, Poland
}

(Received September 3, 1997; revised version April 9, 1998)

\begin{abstract}
Electronic structure and local magnetic moments for the epitaxially grown fcc Fe films included between the $\mathrm{Cu}(001)$ substrate and $\mathrm{Cu}(001)$ covering layers are calculated. The lattice constant of the Fe film is assumed to be equal to that of $\mathrm{Cu}$ homogeneously in the whole film. Interface parts of the Fe film are found to be ordered ferromagnetically, while inner part of the Fe film is obtained as ordered layer-by-layer antiferromagnetically for odd number of layers. For even number of Fe layers the most favourable configuration includes antiferromagnetism of inner layers with two middle layers coupled ferromagnetically.
\end{abstract}

PACS numbers: $75.70 . A k$

\section{Introduction}

Magnetic properties of ultrathin fcc Fe films epitaxially grown on $\mathrm{Cu}(001)$ substrates are a subject of a quite substantial number of experimental and theoretical studies [1-8]. They are of particular interest, because the epitaxially grown fcc Fe films differ in magnetic properties both from the usual bcc phase of iron as well as the high temperature fcc Fe phase. Moreover, it has been established very well both theoretically [1-3] as well as experimentally [4-8] that the surface monolayers of this system are coupled ferromagnetically, while there is the long-lasting controversy concerning the magnetic state of the inner layers of the films: first-principles theoretical approaches suggest that the inner layers are ordered layer-by-layer antiferromagnetically but experimental data show that they are unambiguously paramagnetic at room temperature. It is important, however,

\footnotetext{
*e-mail: wojne@ifpan.edu.pl
} 
that both results do not exclude each other, because - as it has been established with the conversion-electron Mössbauer spectroscopy - the fcc Fe films have an antiferromagnetic state below $T_{\mathrm{N}}=65 \mathrm{~K}$ and above this temperature - they are magnetically disordered [4]. This shows that the fcc Fe(001) films stabilized by the $\mathrm{Cu}$ - substrate in the fcc phase and at a lattice constant close to that of $\mathrm{Cu}$ have an antiferromagnetic ground state - inside the film [6], and the ferromagnetic ordering - at the surface [5]. This means that the role played by $\mathrm{Cu}$ in the considered system is extremely important. Moreover, the first-principles theoretical treatments show also that the results concerning the magnetic properties of the system are very sensitive with respect to the value of the lattice constant [1-3]. In particular, calculations of the electronic structure and the magnetic moment of the local monolayers together with calculation of the local spacing have been performed for fcc Fe films on $\mathrm{Cu}(001)$ [3] and show an enhancement of ferromagnetic moment in the surface layer compared to bulk ferromagnetic fcc $\mathrm{Fe}$, a reduced ferromagnetic moment in the second layer coupled ferromagnetically to the surface layer, and antiferromagnetic order of inner layers.

In our work we calculate the electronic structure and the magnetic moments of the epitaxially grown fcc Fe films included between the $\mathrm{Cu}(001)$ substrate and the $\mathrm{Cu}(001)$ covering layers, with the periodic boundary conditions and the lattice constant assumed to be equal to that of $\mathrm{Cu}(3.61 \AA)$, homogeneously in the whole system. With these assumptions we hope to obtain the most important features of the magnetic properties, which are independent of the very fine particulars of the structure of the system considered. Our calculations were performed for fcc $\mathrm{Fe}$ films of five, six, seven, and eight layers thick. It should be stressed here that the results for inner parts of films with even numbers of Fe layers were not discussed in the previous papers.

The calculations were made self-consistently using the linear muffin-tin orbital (LMTO) method in the relativistic, spin polarized approximation. Spin-orbit coupling was included. The calculations were performed using $273 k$ points in the irreducible part of the Brillouin zone (BZ) for tetragonal lattice (eight, ten, or twelve atoms per unit cell). A sufficiently large number of iterations was carried out to have the total energy converged to an accuracy better than five microrydbergs. The von Barth-Hedin approximation for exchange and correlation was used. All $s, p, d$ electrons were taken into account.

\section{Results}

For five monolayers thick fcc Fe films included between $\mathrm{Cu}(001)$ substrate - simulated by two monolayers of $\mathrm{Cu}$ - and one covering monolayer of $\mathrm{Cu}$, with periodic boundary conditions in direction perpendicular to the film, the results are basically similar to those obtained in Refs. [1] and [2]. Figure 1 shows the local densities of states for all monolayers of the film. It can be seen that first two layers at the interfaces with $\mathrm{Cu}$ are coupled ferromagnetically one to another, but the midplane monolayer is ordered antiferromagnetically with respect to the surfaces. The magnetic moments are 2.46, 2.05, -1.57, 2.05, 2.46 in Bohr magneton units. The magnetic moment of the first layer at both surfaces is enhanced with respect to the moment of the bulk $\mathrm{Fe}$, while the moment of the second layer is reduced. 


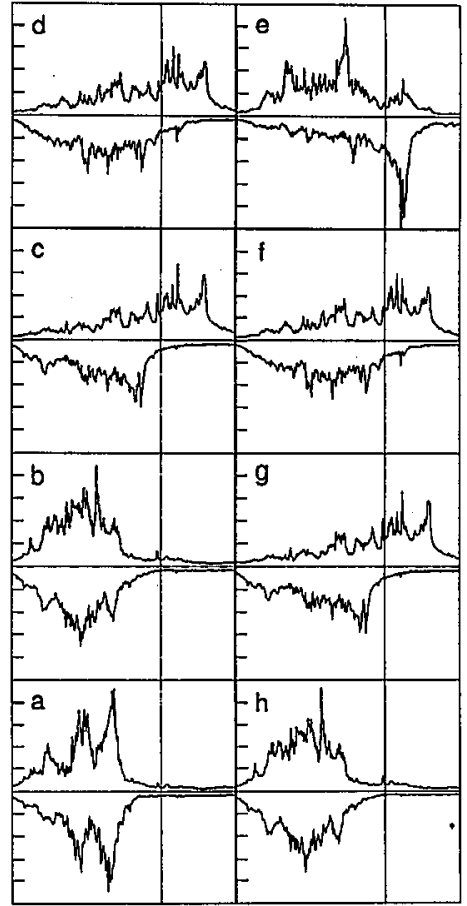

Fig. 1. Density of states (DOS) for the 5-monolayer Fe film with $(++-++)$ magnetic configuration: (a) $\mathrm{Cu}$ (middle), (b) $\mathrm{Cu}$ (interface), (c) $\mathrm{Fe}$ (interface), (d) $\mathrm{Fe}$ (next to interface), (e) $\mathrm{Fe}$ (middle), (f) $\mathrm{Fe}$ (next to interface), (g) $\mathrm{Fe}$ (interface), (h) $\mathrm{Cu}$ (interface). Scale of energy: from $-0.4 \mathrm{Ry}$ to $+0.2 \mathrm{Ry}$; Fermi level corresponds to zero of energy (shown by vertical line). Scale of DOS: from 0 to 50 states/(Ry cell spin) shown by horizontal ticks separated by 10 states/(Ry cell spin). Upper parts - spin up, lower parts - spin down.

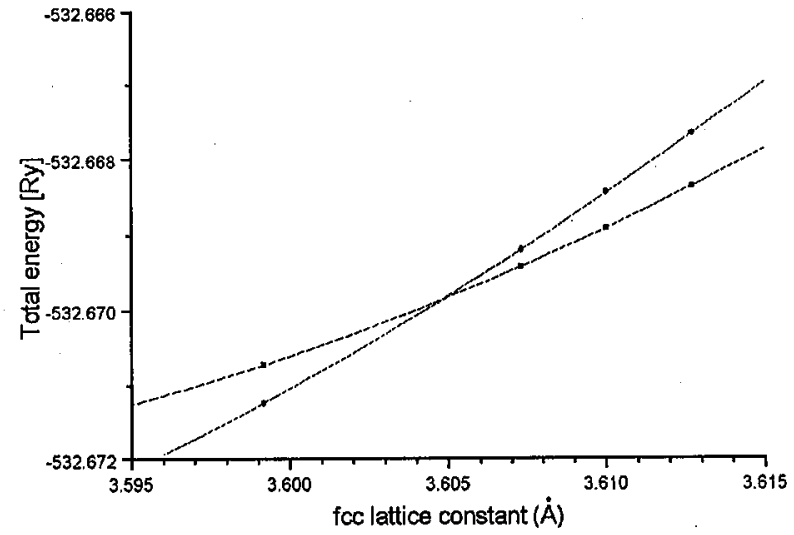

Fig. 2. Total energy for five monoläyers of Fe films. Squares correspond to $(++-++)$ and circles to $(++-+-)$ configurations. Lines are drawn by parabolic fit. 
The value of the central (third) Fe monolayer, which is coupled antiferromagnetically, is reduced even more. The only difference with Refs. [1-3] is in the value of the magnetic moment of the first layer, which is enhanced not so much as in [1-3]. This is the result of different geometry of our system as compared with [1-3]. It is important to note that our result depends strongly on the lattice constant used for the Fe film. If some changes of the lattice constant are allowed, the $(++-+-)$ configuration may correspond to the lower total energy (Fig. 2).

Interesting results are obtained for the fcc Fe film of six monolayers of thickness, included between two monolayers of Cu substrate and two covering monolayers of $\mathrm{Cu}$, also with periodic boundary conditions in the direction perpendicular to the film. The magnetic configuration which is most favourable energetically can be described schematically as the $(++--++)$ configuration. Figure 3 depicts the density of states for all monolayers of such a configuration. The magnetic moments for Fe monolayers are: $2.47,1.99,-1.97,-1.95,2.00,2.48$ (in Bohr magneton units). This result shows that first two layers at the interface with $\mathrm{Cu}$ are coupled ferromagnetically again but two monolayers inside the film are ordered antiferro-
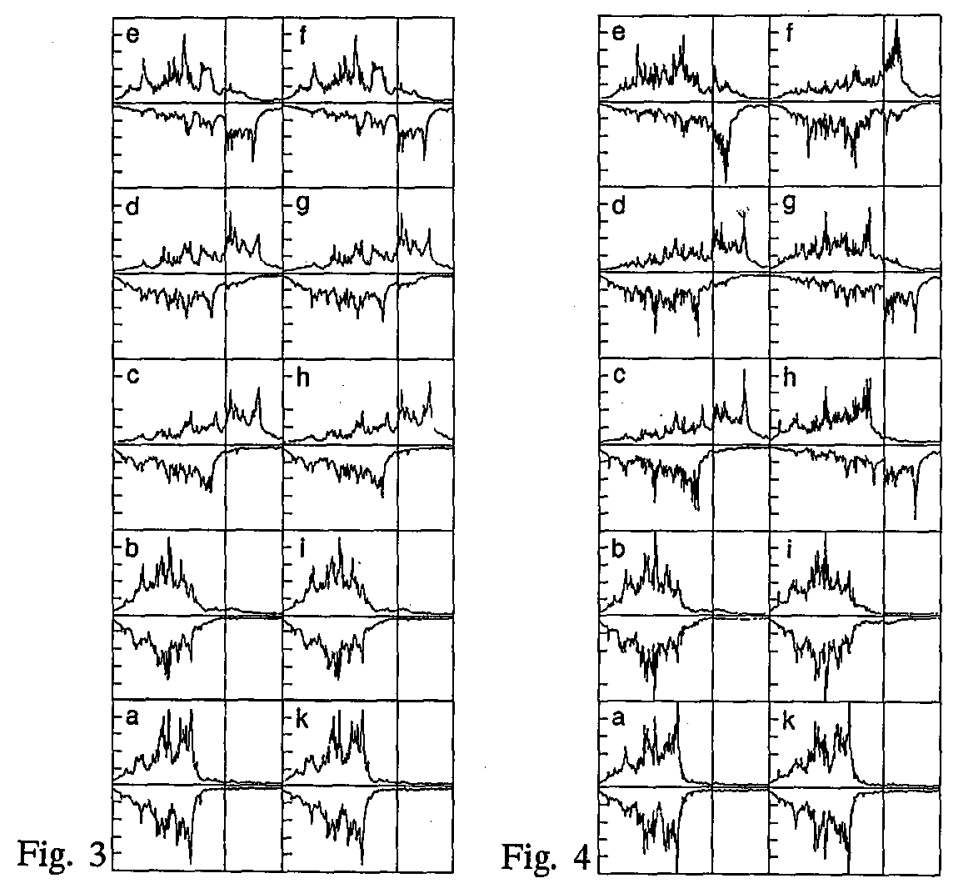

Fig. 3. Density of states for the 6-monolayer Fe film with $(++--++)$ magnetic configuration: (a) $\mathrm{Cu}$ (next to interface), (b) $\mathrm{Cu}$ (interface), (c) $\mathrm{Fe}$ (interface), (d) $\mathrm{Fe}$ (next to interface), (e) Fe (middle), (f) Fe (middle), (g) Fe (next to interface), (h) $\mathrm{Fe}$ (interface), (i) $\mathrm{Cu}$ (interface), (k) $\mathrm{Cu}$ (next to interface). Rest of the caption as for Fig. 1. Fig. 4. Density of states for the 6-monolayer Fe film with $(++-+--)$ magnetic configuration. Rest of the caption as for Fig. 3. 
magnetically with respect to the surfaces, but ferromagnetically one to another. If one wants to get both surfaces of the film magnetized in the opposite direction, the $(++-+--)$ configuration with higher total energy (a difference is 2.28 milirydbergs) has to be considered. Figure 4 shows the corresponding density of states for all monolayers of the film in this case. Now, the magnetic moments for Fe monolayers are: $2.48,2.08,-1.47,1.47,-2.08,-2.48$ (in Bohr magneton units). Again, first two layers at the both surfaces are coupled ferromagnetically, however, now the moments in the middle of the system are strongly reduced. In this case the results obtained are only weakly sensitive with respect to the lattice constant changes (Fig. 5).

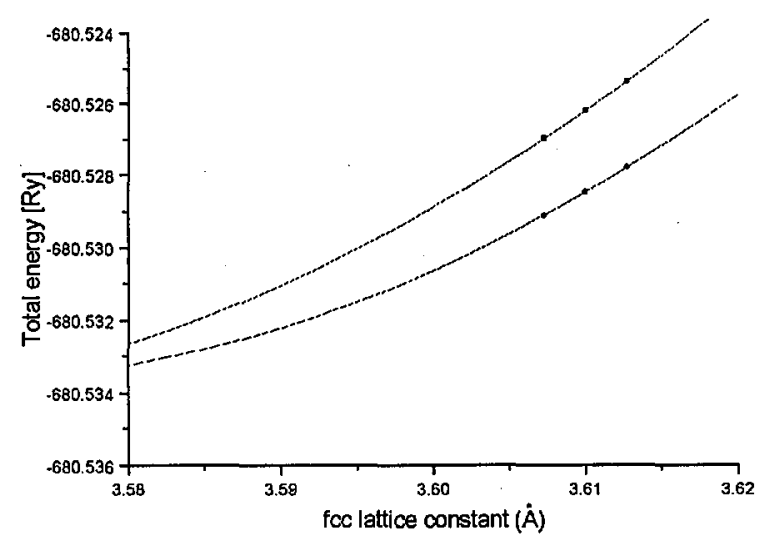

Fig. 5. Total energy for six monolayers of Fe films. Squares correspond to $(++-+--)$ configuration, circles - to $(++--++)$ configuration. Lines are drawn by parabolic fit.

For fcc Fe films of seven monolayers of thickness, included between two monolayers of $\mathrm{Cu}$ substrate and one covering $\mathrm{Cu}$ monolayer, with periodic boundary conditions in the direction perpendicular to the film, the most favourable energetically is the $(++-+-++)$ configuration. Figure 6 gives the density of states for all monolayers in this case and the resulting magnetic moments are: 2.46, 2.04, $-1.54,1.37,-1.54,2.04,2.46$. As previously, first two layers at the interfaces with $\mathrm{Cu}$ are coupled ferromagnetically but now the inner part of the film is ordered layer-by-layer antiferromagnetically. Other configurations e.g. $(+++--++)$ or $(+-+-+-+)$ have the total energy slightly higher (the difference is about $0.79 \mathrm{mRy}$ ). This result shows that for films with bigger but odd number of monolayers and boundary conditions similar for the top surface of the film as for the bottom surface, the antiferromagnetic ordering is expected inside the film. In this case our result depends strongly on the lattice constant used for the Fe film. If some changes of the lattice constant are allowed, the $(+++--++)$ configuration may correspond to the lower total energy, but in this case - for greater lattice constants. 

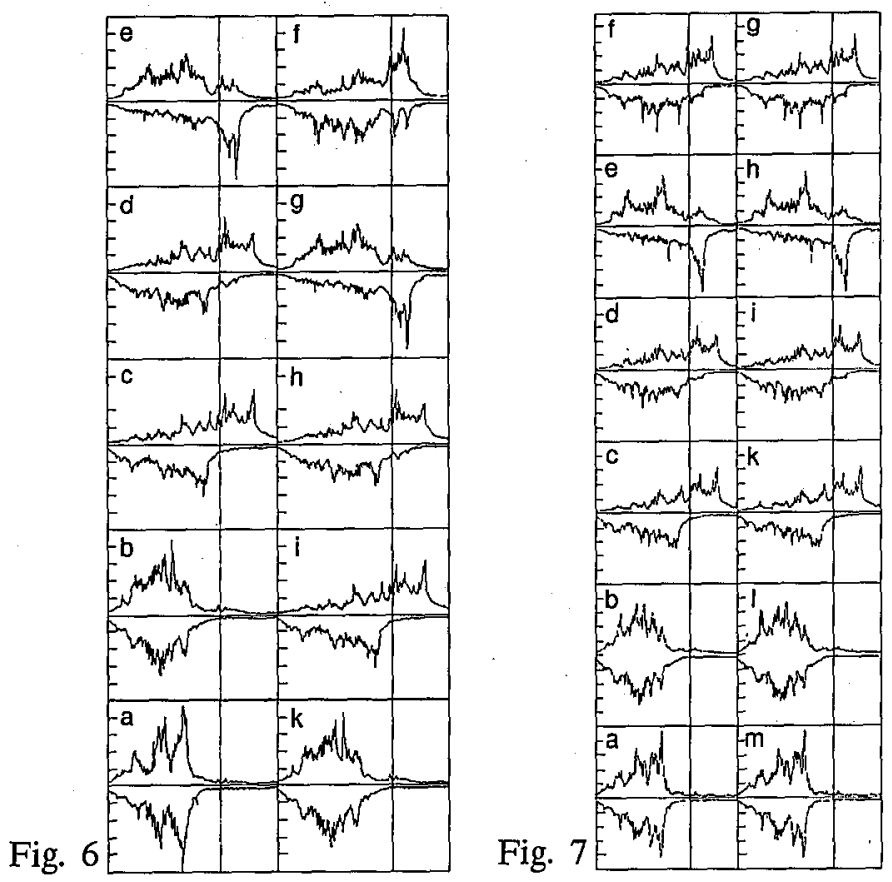

Fig. 6. Density of states for the 7 -monolayer Fe film with $(++-+-++)$ magnetic configuration: (a) $\mathrm{Cu}$ (middle), (b) $\mathrm{Cu}$ (interface), (c) Fe (interface), (d) Fe (next to interface), (e) Fe (next to middle), (f) Fe (middle), (g) Fe (next to middle), (h) Fe (next to interface), (i) $\mathrm{Fe}$ (interface), (k) Cu (interface). Rest of the caption as for Fig. 1.

Fig. 7. Density of states for the 8-monolayer Fe film with $(++-++-++$ ) magnetic configuration: (a) $\mathrm{Cu}$ (middle), (b) $\mathrm{Cu}$ (interface), (c) $\mathrm{Fe}$ (interface), (d) Fe (next to interface), (e) Fe (next to middle), (f) Fe (middle), (g) Fe (middle), (h) Fe (next to middle), (i) $\mathrm{Fe}$ (next to interface), (k) $\mathrm{Fe}$ (interface), (l) $\mathrm{Cu}$ (interface), (m) Cu (middle). Rest of the caption as for Fig. 1.

For the fcc Fe film of eight monolayers of thickness the magnetic configuration which is most favourable energetically is the $(++-++-++)$ configuration. Figure 7 depicts the density of states for all monolayers with such a configuration. The magnetic moments for Fe monolayers are: $2.48,2.05,-1.60,1.67,1.66,-1.61$, $2.05,2.48$ (in Bohr magneton units). The total energy for the $(++-+-+--)$ configuration is slightly higher: a difference is 1.52 milirydbergs - similarly as in the case of six monolayers. The magnetic moments in this case are: $2.47,2.05$, $-1.49,1.40,-1.40,1.49,-2.05,-2.47$ (in Bohr magneton units). Here the results obtained are only weakly sensitive with respect to the lattice constant changes similarly to six-layer case. Our results show clearly that in an even number layer Fe film, the most favourable configuration includes two middle monolayers coupled ferromagnetically!

In all cases discussed above some very small induced moments appear in the $\mathrm{Cu}$ monolayers as the result of proximity effects. 


\section{Conclusions}

The results obtained in this paper support two main features of the considered system: ferromagnetic coupling for first two monolayers at the surfaces of the fcc Fe film and aniferromagnetism inside the system, however, for the even number of $\mathrm{Fe}$ layers the most favourable configuration includes two middle monolayers coupled ferromagnetically. In a nearly symmetric system of $\mathrm{Cu} / \mathrm{Fe} / \mathrm{Cu}$, only odd number of $\mathrm{Fe}$ monolayers can keep both interfaces in ferromagnetic coupling and all inner layers in antiferromagnetic configuration. Less important conclusions are connected with interface effects at the $\mathrm{Cu}$ substrate and $\mathrm{Cu}$ covering, which may modify the magnetic moments. Another conclusion is that for configurations with lowest energies, both surfaces of the fcc Fe film are magnetized parallel. Both surfaces could be magnetized in opposite directions if somehow additional energy is supplied.

Finally, we should admit that energy differences for various configurations considered in this work are very small and dependent on changes of the lattice constant, especially for the odd number of Fe monolayers. Moreover, according to Refs. [1-3] changes of interlayer spacing can also influence the total energy of the system, inducing changes of these configurations which are the most favourable. However, variety of possibilities is large and will be investigated in our next papers.

\section{Acknowledgments}

We would like to thank Dr. Piotr Kociński for cooperation in early stage of this work.

\section{References}

[1] C.L. Fu, A.J. Freeman, Phys. Rev. B 35, 925 (1987).

[2] G.W. Fernando, B.R. Cooper, Phys. Rev. B 38, 3016 (1988).

[3] T. Kraft, P.M. Marcus, M. Scheffler, Phys. Rev. B 49, 11511 (1994).

[4] W.A.A. Macedo, W. Keune, Phys. Rev. Lett. 61, 475 (1988).

[5] J. Thomassen, F. May, B. Feldmann, M. Wuttig, H. Ibach, Phys. Rev. Lett. 69, 3831 (1992).

[6] Dongqi Li, M. Freitag, J. Pearson, Z.Q. Qiu, S.D. Bader, Phys. Rev. Lett. 72, 3112 (1994).

[7] M. Donath, B. Gubanka, F. Passek, V. Dose, 15th Gen. Conf. Cond. Matter Div., EPS, Baveno-Stresa (Italy) 1996, Europhysics Conf. Abstr., Vol. 20A, 1996, p. 143.

[8] M. Matsui, M. Doi, A. Kida, Y. Yamada, J. Appl. Phys. 79, 5883 (1996) (abstract only). 